\title{
From Intermittent to Nonintermittent Behavior in Two Dimensional Thermal Convection in a Soap Bubble
}

\author{
F. Seychelles, ${ }^{1, *}$ F. Ingremeau, ${ }^{1}$ C. Pradere,${ }^{2}$ and H. Kellay ${ }^{1}$ \\ ${ }^{1}$ Centre de Physique Moléculaire Optique et Hertzienne, Université Bordeaux 1, UMR 5798 du CNRS, 351 cours de la Libération, \\ 33405 Talence, France \\ ${ }^{2}$ Laboratoire TREFLE, CNRS UMR 8508, Esplanade des Arts et Métiers, 33405 Talence, France
}

(Received 19 July 2010; published 29 December 2010)

\begin{abstract}
Turbulent thermal convection in half a soap bubble heated from below displays a new and surprising transition from intermittent to nonintermittent behavior for the temperature field. This transition is observed here by studying the high order moments of temperature increments. For high temperature gradients, these structure functions display Bolgiano-like scaling predicted some 60 years ago with no observable deviations. The probability distribution functions of these increments are Gaussian throughout the scaling range. These measurements are corroborated with additional velocity structure function measurements.
\end{abstract}

DOI: 10.1103/PhysRevLett.105.264502

PACS numbers: 47.55.pb, 47.27.- i, 47.55.Hd

The importance of turbulent thermal convection for processes of meteorological, geophysical, or industrial interest has been stressed for over a century. Many experimental and theoretical endeavors have explored this phenomenon at different scales and for different geometries $[1,2]$ in the canonical situation of a fluid enclosed in a container heated from below and cooled at the top [3]. As for three dimensional hydrodynamic turbulence [4-6], the statistical properties of temperature and velocity fluctuations in turbulent thermal convection, a state which can be reached for a high enough temperature difference between the bottom and the top of the container, can also be described by scaling laws [7,8]. Several experiments have been carried out to measure these statistical properties but a number of issues regarding the scaling properties remain unresolved [9]. Recently, the two dimensional version has been put forth using either vertical soap films or soap bubbles $[10,11]$. A detailed examination of the statistical properties of the velocity fluctuations and the density variations $[10,11]$ showed that they displayed scaling laws predicted by Bolgiano and Obukhov for stratified turbulence in the 1950s [7-9]. Such scaling laws have so far been elusive in three dimensional experiments for reasons still debated today $[9,12]$.

We here explore the temperature field in a recently introduced thermal convection cell: half a soap bubble heated from below (see Fig. 1) [11]. This geometry has the advantage of avoiding the presence of side walls and therefore the presence of the large scale circulation often observed when lateral walls are present. By focusing on the structure functions of the temperature field we uncover a transition from an intermittent to a nonintermittent behavior. Our results show that the scaling of these functions switches regimes from the so-called Obukhov-Corrsin-like scaling $[5,6]$ with intermittency at low temperatures to Bolgiano-Obukhov-like scaling without intermittency at higher temperatures. Our results are unique and surprising since previous numerical work indicated the presence of strong intermittency for the temperature field [13]. Intermittency in fluid turbulence is an important problem in hydrodynamics and our experiments bring to light how a simple system evolves from an intermittent to a nonintermittent state.

The convection cell, described previously in [11], consists of a hollow brass ring which is heated using water from a thermostat. A circular groove is engraved on the

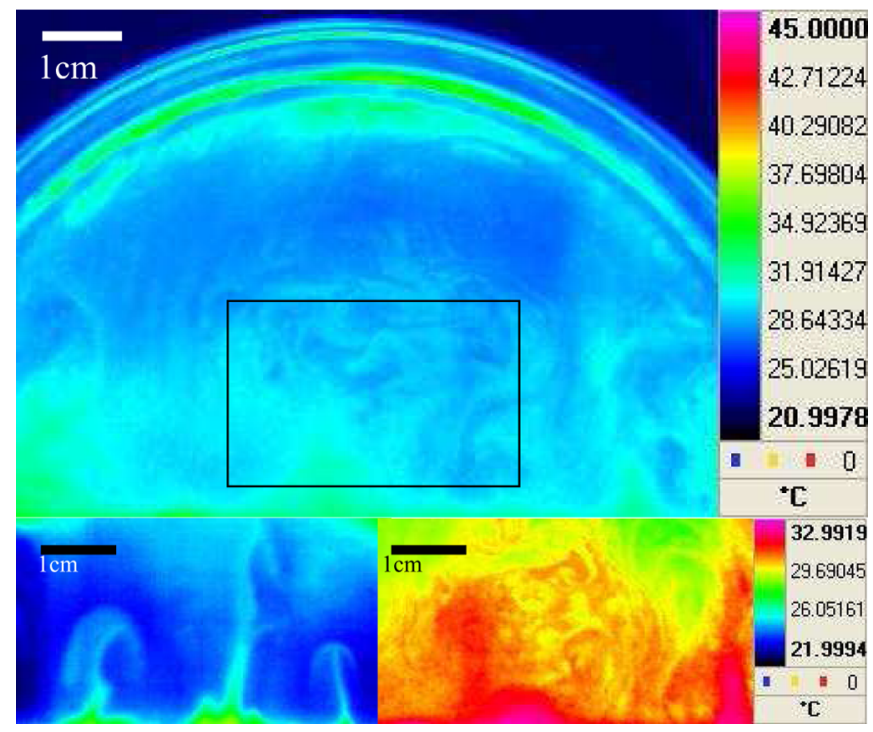

FIG. 1 (color online). Infrared images of the bubble (top $\Delta T=$ $50{ }^{\circ} \mathrm{C}$ ) and a region near the bottom: $\Delta T=21^{\circ} \mathrm{C}$ (bottom left) and $50^{\circ} \mathrm{C}$ (bottom right). The region delimited by a rectangle in the upper image indicates the area covered by the temperature and velocity measurements. The brass ring is located a few millimeters from the bottom of the images. 
upper side of the ring and contains the soap solution ( 0.5 to $2 \%$ detergent in water) which is heated to the desired temperature (up to $85^{\circ} \mathrm{C}$ ). Half a soap bubble, of $12 \mathrm{~cm}$ in diameter, is blown using a straw and the soap solution in the groove. The setup is in a room kept at a constant temperature of $17^{\circ} \mathrm{C}$ with a humidity rate of nearly $75 \%$ near the bubble. The temperature gradient between the bottom and the top of the half bubble $\Delta T$ could be varied up to $55^{\circ} \mathrm{C}$. The temperature measurements used a calibrated 14 bits infrared camera (resolution $256 \times 360$ ) working in the spectral range of 3.6 to $5 \mu \mathrm{m}$ with a sensitivity of $20 \mathrm{mK}$ and an adjustable exposure time set between 0.5 and $1 \mathrm{~ms}$. Additional measurements were carried out using another infrared camera working in the range 1.9 to $5.2 \mu \mathrm{m}$ but equipped with a band pass filter in the range 3 to $4 \mu \mathrm{m}$ with no noticeable changes in the results. Images of the same region (between 100 and 500 images at a rate of 50 or 100 frames/second) were recorded and a home made program was used to calculate temperature differences across different scales $r$. Averaging over the area of interest and over several images allowed us to improve the statistics (between 1 and $2.5 \times 10^{6}$ points were used) and calculate the high order moments of these differences. The temperature field was recorded for periods of up to $10 \mathrm{~s}$ which is greater than the temperature correlation time (of order $0.1 \mathrm{~s}$ ). The error in $r$, introduced by the curved geometry of the bubble, turned out to be less than a few percent over a $1 \mathrm{~cm}$ region. The effect of evaporation was estimated to be small and the lifetime of the bubble, which should decrease with increased evaporation, actually increases by a factor of about 4 when a temperature gradient is imposed indicating that convection is more important than both evaporation and draining by gravity.

Figure 1 shows a full view of the bubble as well as images obtained with the infrared camera in a region near the bottom of the half bubble where the thermal convection is strongest. One can easily identify thermal plumes rising from the bottom of the cell which are clearly visible for the low temperature gradient. The thermal convection becomes more intense as the temperature gradient increases and well-defined thermal plumes are difficult to discern. From such spatial images we extract the temperature difference $\delta T\left(r_{x}\right)=T\left(x+r_{x}\right)-T(x) \quad$ and $\quad \delta T\left(r_{y}\right)=T\left(y+r_{y}\right)-$ $T(y)$ and calculate the $n$th moments as $\left\langle\left|\delta T\left(r_{x}\right)\right|^{n}\right\rangle$ and $\left\langle\left|\delta T\left(r_{y}\right)\right|^{n}\right\rangle$. Here $x$ and $y$ refer to the horizontal and vertical coordinates and the brackets refer to an average over space and time. The temperature structure functions are important quantities in the study of turbulence and different scaling relations have been proposed for their variation versus the scale $r$. In 3D turbulent flows, where Kolmogorov-like scaling is believed to prevail for the low order moments, Obhukov and Corrsin [5,6] generalized the scaling arguments of Kolmogorov to a scalar field like the temperature and used both the energy dissipation rate $\epsilon$ and the scalar dissipation rate $\epsilon_{\theta}$ to predict that the second order structure functions should scale as $\epsilon_{\theta} \epsilon^{-1 / 3} r^{2 / 3}$. Similar scaling arguments can be used, as suggested by Bolgiano and Obukhov [7-9] for stably stratified turbulence, to the case of Rayleigh Benard convection for which the fluid thermal expansion rate $\beta$, the gravity constant $g$, and the dissipation rate $\epsilon_{\theta}$ fix the scaling relation of the second order structure function of the temperature as $\epsilon_{\theta}^{4 / 5}(\beta g)^{-2 / 5} r^{2 / 5}$ [9]. The $n$th order moments are expected to vary as a power law of the separation distance $r$ with an exponent $\zeta_{n}^{T}$ of $n / 5$ in the Bolgiano-Obukhov regime and $n / 3$ for the ObukhovCorrsin regime. To compare the experimental conditions here to their classical counterparts, we estimated the Rayleigh number $\left(\mathrm{Ra}=\beta \Delta T g R^{3} / \nu \kappa\right.$ where $\nu$ and $\kappa$ are the kinematic viscosity and the thermal diffusivity of water) to be between $7 \times 10^{7}$ and $2 \times 10^{8}$ while the Reynolds number $\left(\operatorname{Re}=V_{\text {mean }} R / \nu\right.$ where $V_{\text {mean }}$ is the characteristic horizontal velocity) is estimated to be about 3000 .

The temperature structure functions are displayed in Figs. 2(a) and 2(b) for two different $\Delta T: 21$ and $50{ }^{\circ} \mathrm{C}$. For the low $\Delta T$, Fig. 2(a), the temperature structure functions are roughly isotropic as the values of the differences for the two orthogonal spatial increments $r_{x}$ and $r_{y}$ are
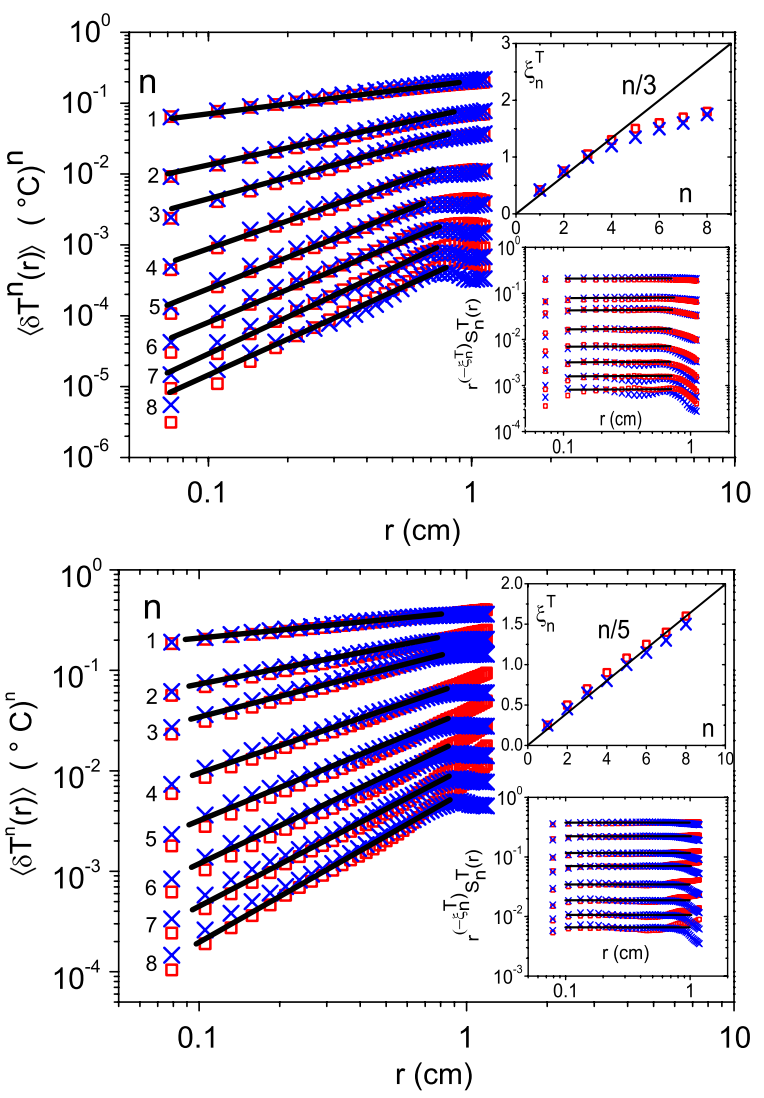

FIG. 2 (color online). Temperature structure functions for $\Delta T=21^{\circ} \mathrm{C}$ (a) $\Delta T=50^{\circ} \mathrm{C}$ (b). The horizontal (squares) and vertical (crosses) components are plotted up to order 8 . The data are shifted by a multiplicative factor ( $x 2$ for $n=3$ up to $x 64$ for $n=8$ ). Insets: compensated moments and scaling exponents. 
similar. These functions display power law scaling for spatial scales between roughly 1 and $10 \mathrm{~mm}$ as the compensated moments show. The scaling exponents vary in a nontrivial manner versus the order $n$ of the moment. This exponent is in agreement with predictions of Obukhov and Corrsin [5,6] for low $n$ in Kolmogorov-like turbulence [4]. However, for higher moments, the exponents deviate from this prediction. The growth is nonlinear versus $n$ which is the hallmark of intermittency. The relation between the higher order moments and the low order ones is nontrivial indicating that the functional shape of the probability distribution functions of the increments varies with $r_{x}$ or $r_{y}$. This behavior is similar to that observed in three dimensional experiments where Bolgiano-like scaling has not been observed so far; rather Obukhov-Corrsin scaling with deviations, just like passive scalar fields in three dimensional hydrodynamic turbulence, is observed $[9,12,14]$.

The high $\Delta T$ results are shown in Fig. 2(b). While the structure functions show isotropy and power law scaling versus $r$, the variation of the exponents versus $n$ turns out to be different from the previous results. Bolgiano-Obukhovlike scaling is observed in the range 1 to $10 \mathrm{~mm}$ as shown in Fig. 2(b) which display the compensated moments as well as the scaling exponents extracted from such an analysis. Estimates of the Bolgiano length scale (above which such a scaling is believed to prevail) give $L_{B} \sim 1 \mathrm{~mm}$ which is in good agreement with the range observed here and in previous experiments using vertical films [10]. The surprising aspect is that a linear variation of the exponents versus $n$ is observed. This linear variation indicates that intermittency is absent. This behavior has been observed for an imposed $\Delta T$ higher than about $35^{\circ} \mathrm{C}$.

Additional insight into this transition comes from an examination of the probability density functions (PDF) of the temperature increments in the range of scales for which power laws are observed. These results are shown in Fig. 3. The horizontal axis has been rescaled by the standard deviation $\sigma\left(=\sqrt{\left\langle|\delta T(r)|^{2}\right\rangle}\right)$ of the temperature increment at the scale $r$ while the vertical axis has been normalized in such a way that the integral of the function is unity. Note that for the small gradient, the PDFs start out as a stretched exponential with an exponent near 0.7 at the small scale end of the scaling range $(1 \mathrm{~mm})$ and end up as an exponential for the large scale end $(9 \mathrm{~mm})$. The PDFs evolve gradually as the scale increases from 1 to $10 \mathrm{~mm}$, indicating a change of the functional shape of the PDF across the scales. For the high $\Delta T$, the PDFs remain roughly Gaussian as the scale changes from 0.9 up to $8 \mathrm{~mm}$. The normalization of the PDFs by $\sigma$ collapses all the PDFs together indicating that they depend solely on the width of the distribution. An examination of the flatness of these PDFs shows that for the low $\Delta T$, the flatness decreases from roughly 10 to 5 as the scale increases from 1 to $10 \mathrm{~mm}$. On the other hand, the flatness for the high $\Delta T$

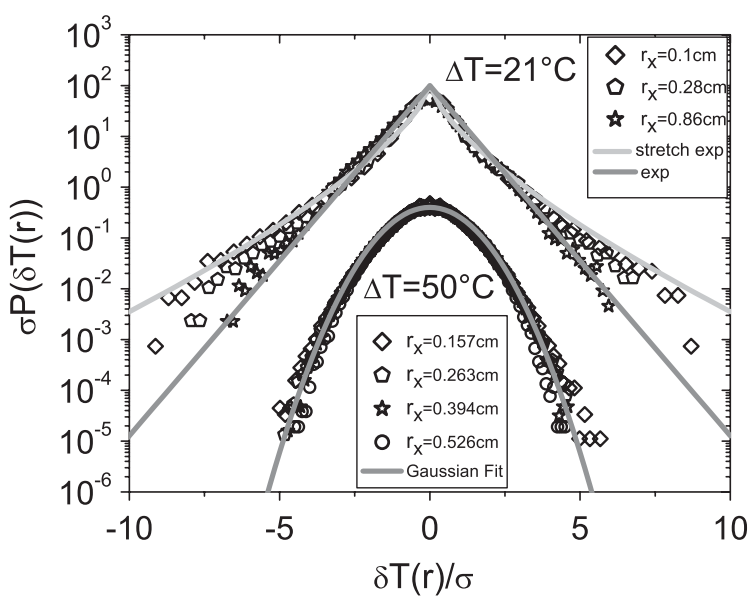

FIG. 3. PDFs of temperature increments for $\Delta T=21{ }^{\circ} \mathrm{C}$ (upper set) $\Delta T=50^{\circ} \mathrm{C}$ (lower set). The axes were rescaled by the standard deviation $\sigma$ of the increments. Solid lines: fits using a stretched exponential function and an exponential function. A Gaussian function is used in the lower set of PDFs.

case remains roughly constant near a value of 2.8 which is not far from the flatness of a Gaussian distribution. These features are at the origin of the dependence of the scaling exponents versus $n$. In short, intermittency of the scaling exponents is associated with the gradual change of the functional shape of the probability density of temperature increments. On the other hand, the absence of intermittency is related to the Gaussian PDFs of the increments all through the scaling range.

To complement these observations, we measured the velocity fluctuations at a single point and constructed the horizontal velocity structure functions of order $n$ as $\left\langle|\delta V(\delta t)|^{n}\right\rangle$ where $\delta t$ is a temporal increment. These measurements use a laser Doppler velocimeter and a soap solution seeded with $1 \mu \mathrm{m}$ sized polystyrene spheres. We record between $5 \times 10^{5}$ and $10^{6}$ points over a total period of time of nearly $100 \mathrm{~s}$ which is greater than the velocity correlation time $(0.2 \mathrm{~s})$. The choice of the horizontal component of the velocity $V$ is justified by the presence over sufficiently long periods of time of a mean flow in this direction, at the location of the measurements, allowing the use of the Taylor frozen turbulence hypothesis to convert $\delta t$ to a scale $r$ as $r=V_{\text {mean }} \delta t$. This hypothesis is not tested here, however. The velocity structure functions are expected to vary as power laws of $r$ with an exponent $\zeta_{n}^{V}=3 n / 5$ following similar arguments as for the temperature in the Bolgiano-Obhukov regime.

These functions are displayed in Figs. 4(a) and 4(b) for the low and high $\Delta T$, respectively. Here again, power laws are obtained for the different moments examined. The variation of the exponents, obtained from an analysis of the compensated moments, is nonlinear for the low $\Delta T$ case and linear for the higher one. The expected $3 n / 5$ variation is shown as a solid line. The scaling range in $r$, determined using the Taylor hypothesis, turns out to be 

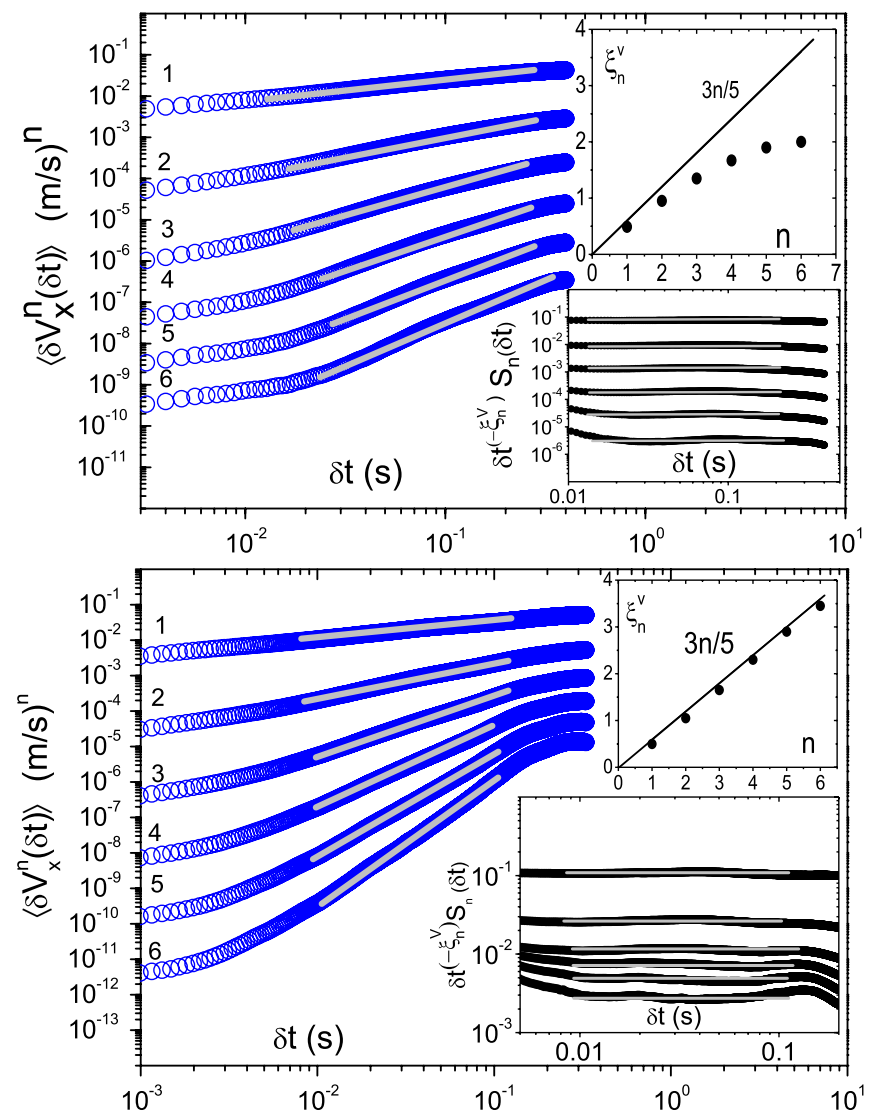

FIG. 4 (color online). $\left\langle|\delta V(\delta t)|^{n}\right\rangle \quad$ for $\quad \Delta T=21{ }^{\circ} \mathrm{C}$ (a) $\Delta T=50{ }^{\circ} \mathrm{C}$ (b). The mean horizontal velocity is $5 \mathrm{~cm} / \mathrm{s}$ in (a) and $8 \mathrm{~cm} / \mathrm{s}$ in (b). Insets: compensated moments and scaling exponents.

similar to that obtained from the temperature structure functions. The variation of the exponents for the low $\Delta T$ case (as well as the flatness of the distributions which decreases from roughly 10 to nearly 3 ) is similar to that obtained by Zhang and $\mathrm{Wu}$ [10]. The agreement between our results and the spatial measurements of Zhang and $\mathrm{Wu}$ seems to validate our use of the Taylor hypothesis for this case. The low temperature gradient statistics therefore show that intermittency is observed for both the velocity and the temperature. For the high $\Delta T$, the scaling exponents vary linearly with $n$ and follow the $3 n / 5$ law in good agreement with the predictions of Bolgiano and Obukhov for the same range of scales $r$ as the temperature. The flatness of the distributions in this case remains roughly constant near a value of 5 . These results are therefore consistent with the temperature measurements and indicate an absence of intermittency for the velocity as well. The Taylor hypothesis in this case has not been tested, however, and the results of Zhang and $\mathrm{Wu}$ did not show an absence of intermittency in their rectangular cells for similar temperature gradients. Our results, even though consistent with the temperature measurements, would need additional confirmation. We noted by examining the PDFs of velocity differences that their evolution with $\Delta T$ is less convincing than that of the temperature: while a better collapse can be achieved for the high $\Delta T$ case, the PDFs are not Gaussian and show roughly exponential tails.

In conclusion, our novel quasi-two dimensional convection cell allows for a detailed study of the statistical properties of temperature fluctuations in turbulent thermal convection. These properties show that a transition from an intermittent state to a nonintermittent one occurs as the temperature gradient increases. Bolgiano-Obukhov-like scaling with no intermittency is recovered for the high gradient case. Our results raise fundamental questions about the role of lateral walls and the ensuing large scale circulation often observed in traditional convection cells as well as the role of thermal plumes in setting the properties of temperature fluctuations in turbulent thermal convection.

This work was supported by Grant "Cyclobulle" from the ANR.

*Present address: FAST, U. Paris Sud, Rue du Belvédère, 91405 Orsay, France.

[1] L. P. Kadanoff, Phys. Today 54, No. 8, 34 (2001).

[2] E. D. Siggia, Annu. Rev. Fluid Mech. 26, 137 (1994).

[3] G. Ahlers, Physics 2, 74 (2009).

[4] A. N. Kolmogorov, Dokl. Akad. Nauk SSSR 30, 299 (1941).

[5] S. Corrsin, J. Appl. Phys. 22, 469 (1951).

[6] A. M. Obukhov, Izvestiia Akademii Nauk SSSR, Ser. Geog. Geofiz 13, 58 (1949).

[7] R. Bolgiano, J. Geophys. Res. 64, 2226 (1959).

[8] A. M. Obukhov, Sov. Phys. Dokl. 4, 61 (1959).

[9] D. Lohse and K.-Q. Xia, Annu. Rev. Fluid Mech. 42, 335 (2010).

[10] J. Zhang and X.L. Wu, Phys. Rev. Lett. 94, 234501 (2005); J. Zhang, X. L. Wu, and K.-Q. Xia, Phys. Rev. Lett. 94, 174503 (2005).

[11] F. Seychelles et al., Phys. Rev. Lett. 100, 144501 (2008).

[12] C. Sun, Q. Zhou, and K.-Q. Xia, Phys. Rev. Lett. 97, 144504 (2006).

[13] A. Celani, A. Mazzino, and M. Vergassola, Phys. Fluids 13, 2133 (2001); A. Celani et al., Phys. Rev. Lett. 88, 054503 (2002).

[14] Z. Warhaft, Annu. Rev. Fluid Mech. 32, 203 (2000). 